\title{
Mendel for the modern era
}

The genome of the model genetic organism Pisum sativum, or pea plant, links nineteenth-century genetics to twenty-first-century genomics, serving as a symbol of how far the genetics field has developed and how greatly technologies have advanced. Almost every student's introduction to genetics currently involves learning Mendel's laws; we envision that genomics and genome sequencing will become just as foundational in the education of future geneticists.

G enetics surrounds us. It is everywhere in the popular media. Increasingly, members of the public are being made aware of issues relating to genomic technologies, whether through having their DNA sequenced through companies such as 23andMe or Ancestry.com, hearing about reports of genome-edited babies or debating how genetically modified crops should be labeled. An interested and engaged public is a positive thing, and we support educational endeavors to promote genetic literacy.

The basic principles of inheritance and independent segregation were worked out through Gregor Johann Mendel's meticulous study of the pea plant in the gardens of Brno in the 1850s and 1860s. Generations of students have learned about dominant and recessive traits through the examples of pea plant height, and pea pod or seed color and shape. The simple laws elucidated by Mendel are experimentally analyzed in classrooms worldwide. Although genetic analysis has become orders of magnitude more sophisticated, Mendel and his humble pea plants are a great guide and entry point into the study of inheritance.

Although most genetics students become familiar with the traits of the pea-including green versus yellow and wrinkled versus smooth, often placed within the ordered Punnett square-as their first foray into genetic study, we believe that a simultaneous, complementary introduction to genomics would also be appropriate.
A basic understanding of what a genome is and how it operates, along with a sense of the complexity and sheer amount of information that genomes hold, is important to teach as early as possible. When public policy is being shaped around the privacy of individuals' genetic data, regulation of gene-edited or genetically modified agricultural products, and guidelines for gene-based therapies to treat diseases, it is crucial for the public to have a basic working knowledge of genetics and genomics. In addition, with the increasing interest in the direct-to-consumer genetic testing used by individuals to find out more about their ancestry, people should understand what those tests are reporting and, more importantly, what their limitations are. This understanding would often require a deeper knowledge of population genetics, but basic principles, from Mendel to genome sequencing, would aid in interpretation.

For example, knowing about the laws of segregation and independent assortment would help people put the understanding of family disease risk variants (how your DNA relates to that of your parents or siblings) into context. Being familiar with concepts of recombination and inheritance would enrich understanding and interpretation of ancestry information. This understanding would also help reduce hype and avoid over-interpretation of genetics findings. Having a fundamental understanding of genetics principles, stemming from Mendel and extending into the genomics era, would empower patients or people getting their DNA sequenced by commercial companies to be better informed and less likely to misinterpret the results.

Although education in genetics is important, we believe that the genomics era has ushered in the need for having a working knowledge of central concepts related to genome biology. Ideally, fundamentals such as the size of the genome, and its linear composition of DNA organized compactly into higher-order chromosomes, would be widespread knowledge. Where genes reside and how they operate from the genome are, of course, still being worked out on the research front. However, the broader concepts, and the links between single genes the whole genome, should be appreciated by a general audience.

Consequently, we are excited to publish the genome sequence of Mendel's pea plant. Although the individual genes and sequences of Mendel's seven original traits have been known for a while, we believe that the genome sequencing of the pea plant represents a symbolic milestone for genetics, bringing the foundational experimental studies in basic models into the modern sequencing era. We hope that Gregor Mendel would approve.

Published online: 2 September 2019 https://doi.org/10.1038/s41588-019-0501-0 